\title{
Relación entre la conducta térmica y el sexo de una población de Pleurodema thaul (Amphibia: Leiuperidae) provenientes de la comuna de Antuco, región del Biobío
}

\author{
Relationship between thermal behavior and sex of a population of Pleurodema thaul \\ (Amphibia: Leiuperidae) of the commune of Antuco, Biobío region
}

\begin{abstract}
Nicza Alveal1,2*, Rafael Salinas ${ }^{1}$ \& Helen Díaz Páez ${ }^{1}$
${ }^{1}$ Laboratorio de Ecofisiología y Conservación de Herpetozoos, Departamento de Ciencias Básicas, Escuela de Educación, Universidad de Concepción, Campus Los Ángeles, Casilla 341, Los Ángeles, Chile.

2Programa de Doctorado en Sistemática y Biodiversidad, Departamento de Zoología, Facultad de Ciencias Naturales y Oceanográficas, Universidad de Concepción, Casilla 160-C, Concepción, Chile.

*Email: nicza7@gmail.com
\end{abstract}

\section{RESUMEN}

Chile es un país que presenta una variedad de ambientes térmicos en los cuales residen diversas especies de anfibios. Para éstos, es importante la temperatura ambiental ( $\mathrm{Ta}$ ) ya que incide sobre su temperatura corporal (Tc). Debido a la alta plasticidad fenotípica de este taxa, la temperatura de aclimatación a la cual son expuestos en condiciones de cautiverio, puede influir en sus respuestas térmicas. Además, el tamaño y/o masa corporal pueden influir sobre dicha conducta, y por ende, el dimorfismo sexual podría diferenciar la Tc. Basado en lo anterior, Pleurodema thaul es un sujeto de estudio óptimo para evaluar el comportamiento térmico ya que presenta dimorfismo sexual en el tamaño corporal y una fuerte influencia de la temperatura de aclimatación sobre la conducta. Para analizar esta asociación se colectaron individuos en la comuna de Antuco (Región del Biobío) y se sometieron a dos temperaturas de aclimatación $\left(10^{\circ}\right.$ y $\left.20^{\circ} \mathrm{C}\right)$. Se comparó su tamaño, masa, Tc y Temperatura seleccionada $\left(T_{\text {see }}\right)$ Los resultados muestran que en ambientes térmicamente homogéneos $P$. thaul es una especie tigmotérmica con una conducta termoconformista. La temperatura de aclimatación no presentó un efecto significativo sobre su comportamiento y seleccionaron un amplio rango térmico con una media sobre los $24^{\circ} \mathrm{C}$ donde no se evidenciaron diferencias significativas entre los sexos.

Palabras clave: Anfibios, dimorfismo sexual, temperatura corporal.

\section{ABSTRACT}

Chile is a country that presents a variety of thermal environments under which diverse species of amphibians reside. For these, the environmental temperature $(\mathrm{Ta})$ is important since it affects their body temperature (Tb). Due to the high phenotypic plasticity of this taxa, the aclimation temperature to which they are exposed in captive conditions can influence their thermal responses. In addition, body size and/ or mass may influence such behavior, and therefore, sexual dimorphism may differentiate Tc. Based on the above, Pleurodema thaul is an optimal object of study to evaluate thermal behavior since it presents sexual dimorphism in body size, and a strong influence of acclimation temperature on behavior. To analyze this association, individuals were collected in the commune of Antuco (Biobío Region) and subjected to two acclimation temperatures $\left(10^{\circ}\right.$ and $\left.20^{\circ} \mathrm{C}\right)$. Their size, mass, Tb and selected temperature $\left(\mathrm{T}_{\mathrm{sel}}\right)$ were compared. The results show that in thermally homogeneous environments $P$. thaul is a tigmothermal species with a thermo-conformist behavior. The acclimation temperature did not present a significant effect on its behavior and they selected a wide thermal range with an average over $24^{\circ} \mathrm{C}$ where there were no significant differences between the sexes.

Keywords: Amphibians, body temperature, sexual dimorphism. 


\section{INTRODUCCIÓN}

La relación del clima y la biología térmica en anfibios es fundamental para su éxito evolutivo y ecológico (Gilchrist 1995, Pörtner 2002). Los cambios en la temperatura ambiental $(\mathrm{Ta})$ de un determinado hábitat ejercen incidencias directas sobre los individuos e influyen a nivel fisiológico y conductual (Huey 1982, 1991). De esta manera, los factores físicos más importantes que caracterizan cada tipo de clima, son determinantes para la distribución espacial y temporal de los anfibios (Huey 1991).

Las variaciones de Ta y su asociación a las conductas térmicas en anfibios presentes en Chile han sido materia de diversos estudios (Iturra-Cid \& Ortiz 2010, Iturra-Cid et al. 2014, Alveal et al. 2016, Vidal et al. 2017). Para P. thaul IturraCid y colaboradores (2014) determinaron un comportamiento termoconformista en condiciones naturales y de laboratorio, indicando que la especie posee una conducta tigmotérmica en períodos de inactividad. Además bajo la exposición a dos temperaturas de aclimatación $\left(10^{\circ} \mathrm{C}\right.$ y $\left.20^{\circ} \mathrm{C}\right)$ las temperaturas seleccionadas $\left(T_{\text {sel }}\right)$ se ven afectadas de manera positiva, es decir, frente a una temperatura de aclimatación mayor, los individuos de $P$. thaul prefieren temperaturas mayores (Iturra 2010). Estos antecedentes, refuerzan la idea de que la alta dependencia que $P$. thaul tiene sobre el ambiente térmico, puede imponer un grave problema para estos individuos a la hora de exponerse a un cambio ambiental drástico.

Dentro de los variados estudios desarrollados en animales, se ha comenzado a enfatizar cómo la masa corporal influye sobre las características fisiológicas de los mismos en aspectos tales como el comportamiento, la reproducción, forrajeo y/o crecimiento (Angilletta et al. 2002). Sin embargo se ha desestimado la influencia que pudieran tener las bases de la termodinámica referidas a la asociación de temperatura y masa. Principios elementales como las propiedades de calor específico señalan que de manera general la capacidad térmica varía de un cuerpo a otro independiente de que estén hechos del mismo material, dos cuerpos pueden tener distintas capacidades térmicas, pues sus masas pueden ser diferentes (Riveiro \& Alvarenga 1998). Consecuentemente con el efecto que juega la inercia térmica al momento de mantener mecanismos termorregulatorios conductuales o fisiológicos (Pough \& Gans 1982), se encuentra el dimorfismo sexual que presenta $P$. thaul asociado al tamaño corporal y masa (hembras más grandes que los machos) (Cei 1962, Iturra-Cid 2010, Francino 2011) por lo que se espera que existan diferencias significativas a nivel intersexual en cuanto a sus Tc y que los machos, por presentar un tamaño menor, prefieran y mantengan temperaturas corporales mayores que las hembras (Zug et al. 2001), ya que individuos de menor tamaño intercambian energía térmica con el ambiente de forma más rápida que los que tienen tamaño mayor, implicando un aumento de la deshidratación y consiguiente ascenso de la temperatura corporal (Zug et al. 2001, Sanabria et al. 2003, Sinervo et al. 2010).

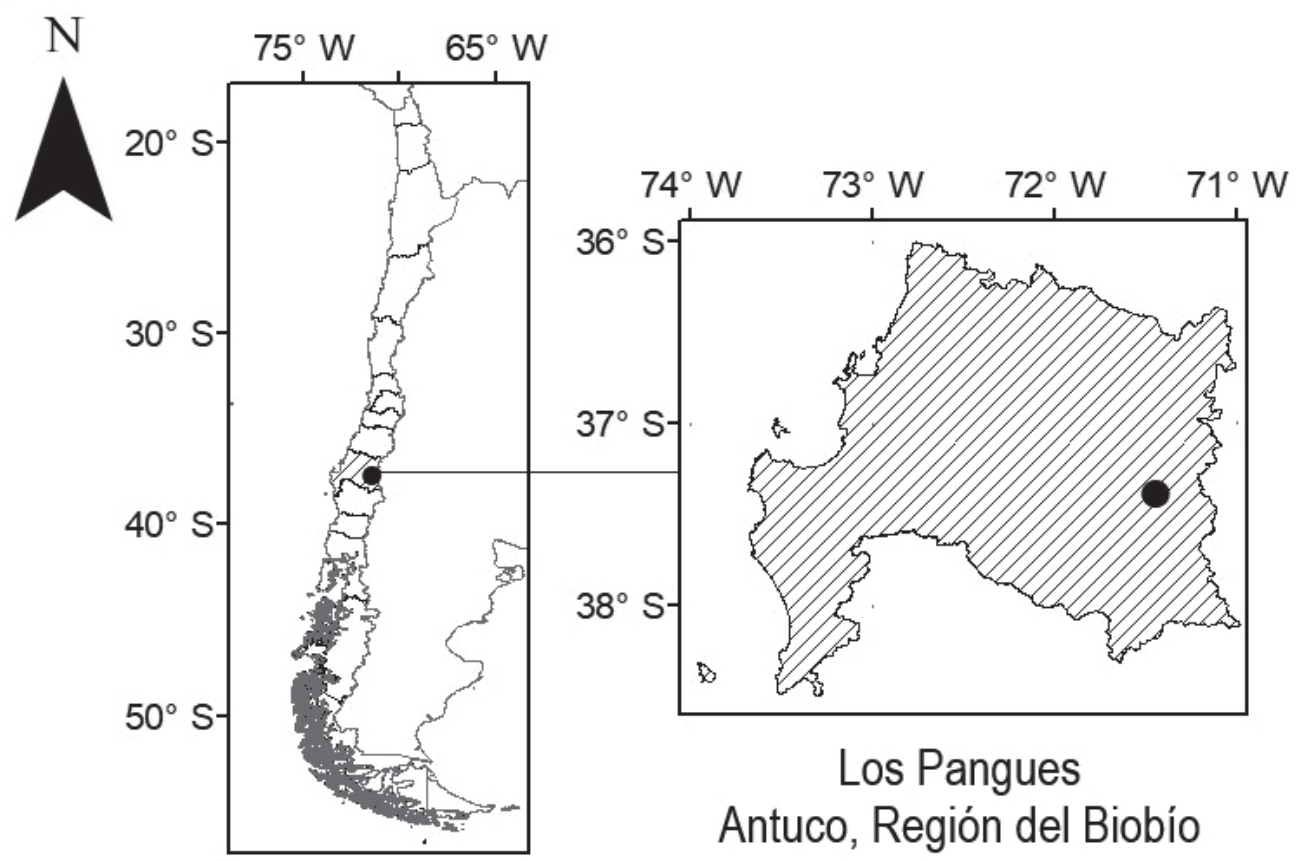

FiguRA 1. Ubicación geográfica del área de captura de P. thaul. / Geographical location of the P. thaul capture area. 


\section{MATERIALES Y MÉTODOS}

\section{ÁREA DE ESTUdIO}

Se colectaron un total de 20 individuos en el sector los Pangues (372' $38^{\prime \prime} \mathrm{S}$; $71^{\circ} 26^{\prime} 16^{\prime \prime} \mathrm{O}, 1024 \mathrm{msnm}$ ) ubicado en la comuna de Antuco, Región del Biobío (Fig. 1), para lo cual se contó con la autorización del Servicio Agrícola Ganadero (Resolución № 9411/2014). Las condiciones climáticas del área corresponden a climas templados de altura, caracterizado por una temperatura variable estacional y diariamente (CONAF 1993). La temperatura media anual registrada es de $6,8^{\circ} \mathrm{C}$ cuyas temperaturas promedio extremas fluctúan entre los $-0,9^{\circ} \mathrm{C}$ (la mínima) y los $14^{\circ} \mathrm{C}$ (la máxima) anual.

\section{OBTENCIÓN DE DATOS DE CAMPO}

La captura se llevó a cabo en forma manual durante la temporada de primavera 2014 a través de la técnica de búsqueda de refugio y encuentro visual. Para la manipulación de los especímenes se utilizaron las medidas establecidas en el "Protocolo para el control de enfermedades infecciosas de Anfibios durante estudios de campo (Lobos et al. 2011).

Cada individuo colectado fue sexado mediante la observación de los caracteres sexuales externos. Además se registró su temperatura corporal de campo y la temperatura del sustrato $\left(T_{s}\right)$ mediante termómetro laser (Fluke 62 mini IR thermometer, $\left.{ }^{\circ} \mathrm{C}\right)$, la temperatura del aire $\left(\mathrm{T}_{\mathrm{a}}\right)$ con termómetro digital (Checktempt $0.1^{\circ} \mathrm{C}$ de precisión), masa corporal mediante balanza digital OHAUS YA-100 de precisión 0,01 g y las medidas de longitud Hocico-cloaca mediante un Pie de metro digital Mitutoyo 6" de precisión 0,01 mm (Cei 1962).

Cada individuo se colocó en bolsas herméticas, para posteriormente ser transportados en cajas de pequeño tamaño habilitadas (con orificios de respiración y papel absorbente humedecido) como contenedor individual y así evitar algún daño físico o la muerte del animal. Finalmente fueron trasladados dentro del mismo día de su captura al laboratorio de Ecofisiología de Herpetozoos del Departamento de Ciencias Básicas de la Universidad de Concepción.

\section{CAUTIVERIO Y ACliMATACIÓN}

En laboratorio, los anfibios capturados fueron mantenidos en condiciones controladas utilizando dos temperaturas de aclimatación $\left(10^{\circ} \mathrm{C}\right.$ y $\left.20^{\circ} \mathrm{C}\right)$ y un fotoperíodo de 12 horas luz y 12 horas oscuridad que se relacionan con las condiciones naturales de esta especie en terreno. Se dispuso de grupos compuestos por dos individuos en 10 acuarios $(30 \mathrm{~cm}$ largo $\times 20 \mathrm{~cm}$ de alto $\times 15$ de ancho). Se utilizó rociadores y recipientes con agua para conservar las condiciones adecuadas de humedad y evitar la deshidratación de los organismos. Estos fueron alimentados principalmente con tebos (Chilecomadia moorei), mosca de la fruta (Drosophila melanogaster) y tenebrios (Tenebrio molitor).

\section{OBTENCIÓN DE DATOS LABORATORIO}

Los especímenes fueron mantenidos en aclimatación bajo dos patrones térmicos $\left(10^{\circ} \mathrm{Cy} 20^{\circ} \mathrm{C}\right)$. Cada período de aclimatación consistió de 15 días, donde la temperatura se mantuvo constante $\left( \pm 1^{\circ} \mathrm{C}\right)$. Durante este período, se obtuvieron los registros térmicos (Ts, Tc y Ta) y corporales (masa y tamaño) de cada ejemplar en condiciones de cautiverio.

Para determinar la $T_{\text {sel, }}$ se utilizó un gradiente térmico conformado de una estructura de metal termo-sellada de 195 $\mathrm{cm}$ de largo, por $60 \mathrm{~cm}$ de ancho y $56 \mathrm{~cm}$ de alto dividido en cuatro carriles de $15 \mathrm{~cm}$ aproximadamente separados por una malla metálica que permite el flujo de calor de forma homogénea entre los diferentes carriles. La superficie consistió en una base de material de acrílico transparente cubierta con gravilla. Bajo esta superficie se colocó un sistema de humedad mediante la conexión de recipientes plásticos con agua para evitar la deshidratación de los individuos. La tapa del gradiente consistió en una superficie de material de acrílico transparente, para evitar que el calor se disipe fuera del sistema. Además, bajo esta cubierta se instaló un sistema de fotoperíodo a fin de mantener las condiciones instauradas en el cautiverio.

Para establecer el gradiente térmico, se utilizó una estufa eléctrica en un extremo y una bomba de refrigeración en el extremo opuesto. El rango promedio de temperaturas del sistema comprendió desde los $8,80^{\circ} \mathrm{C} \pm 1,92$ a los $28,89^{\circ} \mathrm{C} \pm 2,60$. La toma de datos se realizó simultáneamente con cuatro individuos, uno en cada carril. Los animales se colocaron en el centro de cada carril en la mañana y luego de cinco horas se registró la $\mathrm{T}_{\text {sel }}$ de cada uno y el rango del gradiente térmico. Este mismo procedimiento se repitió a las seis y siete horas de haber iniciado el experimento. La media de esos tres valores corresponde a la $T_{\text {sel }}$ de los organismos (Castañeda et al. 2004).

\section{PLAN de ANÁLISIS}

El análisis de los datos obtenidos se realizó mediante estadística descriptiva y se realizaron los test no paramétricos mediante los software estadísticos SPSS versión 13 e InfoStat/E versión 2012 (Di Rienzo et al. 2012).

Se utilizaron los test no paramétricos: Kruskal-Wallis para observar diferencias significativas entre los distintos sexos, y test de Wilcoxon para dilucidar el efecto de la temperatura de aclimatación sobre los parámetros térmicos ( $\left.T c, T_{\text {sel }}\right)$ y en la morfología (tamaño y masa corporal).

Para corroborar el comportamiento térmico y la influencia de la morfología en la temperatura corporal se efectuó una 
predicción con el análisis de regresión lineal múltiple (Draper \& Smith 1998) mediante el software Statistica versión 10.

\section{RESULTADOS}

La especie posee un notorio dimorfismo, el que se evidencia tanto en tamaño como en masa. Las hembras son notoriamente mayores ( $>46 \mathrm{~mm}$ ) que los machos $(>36 \mathrm{~mm}$ ) a ambas temperaturas de aclimatación $\left(\mathrm{H}_{10^{\circ} \mathrm{C}}=264,96\right.$; $\mathrm{P}=0,0001$ y $\mathrm{H}_{20^{\circ} \mathrm{C}}=141,78 ; \mathrm{P}=0,0001$ ), con un rango mucho más amplio en las hembras (Tabla 1).

Los resultados obtenidos durante el terreno muestran que la Tc presenta una media similar en las hembras $\left(7,09 \pm 5,29^{\circ} \mathrm{C}\right)$ $y$ en los machos $\left(7,71 \pm 5,14^{\circ} \mathrm{C}\right)$ sin evidenciar diferencias significativas entre sexos $(\mathrm{H}=0,17 ; \mathrm{P}=0,67)$.

La Tc durante la aclimatación a $10^{\circ} \mathrm{C}$ mostró que los machos alcanzaron una media superior a los $10^{\circ} \mathrm{C}$ muy cercana respecto a la de las hembras, sin exhibir diferencias significativas entre los dos sexos $(H=3,49 ; \mathrm{P}=0,0614)$, en cambio a los $20^{\circ} \mathrm{C}$ la Tc resultó ser significativamente mayor en los machos ( $\mathrm{H}=12,86 ; \mathrm{P}=0,0003)$ (Tabla 1$)$.

Los resultados muestran (Tabla 1 ) que en situaciones controladas mediante la utilización de un gradiente de temperatura entre los $8,8 \pm 1,92^{\circ} \mathrm{C}$ a $38,89 \pm 2,6^{\circ} \mathrm{C}$, la $\mathrm{T}_{\text {sel }}$ presentó una media mayor en las hembras que en los machos (Fig. 2) durante las dos temperaturas de aclimatación. Sin embargo, estas diferencias entre los sexos no fueron significativas (aclimatación $10^{\circ} \mathrm{C}$ : $\mathrm{H}=0,02 \mathrm{P}=0,88$; aclimatación $\left.20^{\circ} \mathrm{C}: \mathrm{H}=2,17 \mathrm{P}=0,14\right)$.

Así mismo, aun cuando se observaron variaciones en las medias de $T_{\text {sel }}$ a diferentes temperaturas de aclimatación, el test de Wilcoxon indica que no son significativas $\left(Z_{\text {machos }}=1,81\right.$ $\mathrm{P}=0,07 ; \mathrm{Z}_{\text {Hembras }}=1,29 \mathrm{P}=0,19$ ), desechando la idea de un posible efecto de la temperatura de aclimatación sobre este parámetro.

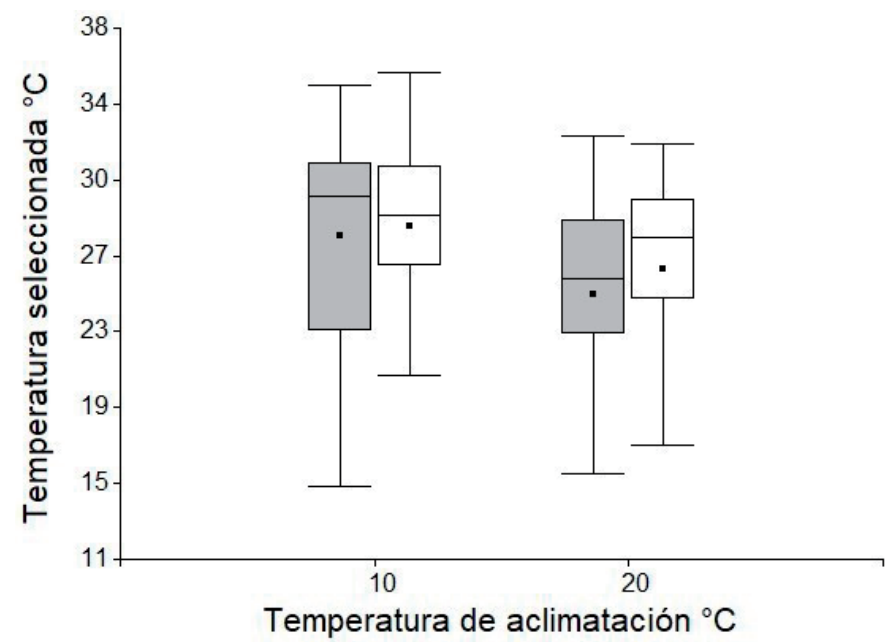

Figura 2. Temperatura seleccionada $\left(T_{\text {sel }}\right)$ para machos (en gris) y hembras (en blanco) de $P$. thaul para la aclimatación de $10^{\circ} \mathrm{C}$ y $20^{\circ} \mathrm{C}$. / Selected temperature $\left(\mathrm{T}_{\text {sel }}\right)$ for males (grey) and females (white) of $P$. thaul for acclimation to $10^{\circ} \mathrm{C}$ and $20^{\circ} \mathrm{C}$.

TABLA 1. Medidas resumen de: Masa corporal, Tamaño corporal, Tc: Temperatura corporal $\mathrm{T}_{\text {sel }}$ Temperatura seleccionada, para los distintos sexos y diferentes temperaturas de aclimatación. / Measures summary of: Body mass, Body size, Tb: Body temperature, $\mathrm{T}_{\text {sel }}$ : Selected temperature, for different sexes and different acclimation temperatures.

\begin{tabular}{lcccc}
\hline Sexo & \multicolumn{2}{c}{ Machos } & \multicolumn{2}{c}{ Hembras } \\
\hline Aclimatación & $10^{\circ} \mathrm{C}$ & $20^{\circ} \mathrm{C}$ & $10^{\circ} \mathrm{C}$ & $20^{\circ} \mathrm{C}$ \\
\hline Masa (g) media \pm D.E. & $4,77 \pm 0,89$ & $4,51 \pm 0,85$ & $9,99 \pm 2,06$ & $9,29 \pm 2,22$ \\
(Rango) & $(3,10-6,40)$ & $(3,10-6,90)$ & $(4,30-14,20)$ & $(3,80-13,60)$ \\
Tamaño (mm) media \pm D.E. & $37,00 \pm 1,98$ & $36,74 \pm 1,94$ & $46,56 \pm 3,20$ & $46,39 \pm 3,20$ \\
(Rango) & $(33,10-41,23)$ & $(32,38-40,02)$ & $(34,62-52,69)$ & $(35,38-53,09)$ \\
Tc $\left({ }^{\circ} \mathrm{C}\right)$ media \pm D.E. & $10,76 \pm 1,10$ & $22,56 \pm 1,29$ & $10,55 \pm 1,16$ & $22,06 \pm 1,41$ \\
$($ Rango) & $(9,00-15,40)$ & $(19,80-27,80)$ & $(9,10-15,30)$ & $(19,60-27,70)$ \\
T $\left._{\text {sel }}{ }^{\circ} \mathrm{C}\right)$ media \pm D.E. & $27,51 \pm 5,86$ & $24,65 \pm 4,81$ & $28,00 \pm 5,43$ & $25,89 \pm 5,70$ \\
$($ Rango $)$ & $(15,07-35,03)$ & $(12,57-32,50)$ & $(15,80-36,70)$ & $(13,17-32,23)$ \\
\hline
\end{tabular}


El cálculo de la pendiente de regresión lineal múltiple en condiciones de campo para machos $\mathrm{Tc}_{\text {machos }}=0,719+1,0061 \mathrm{~T}_{\mathrm{s}}$ - 0,0491T (Fig. 3) y hembras $T c_{\text {hembras }}=5,1289+1,3497 \mathrm{~T}_{\mathrm{s}}-$

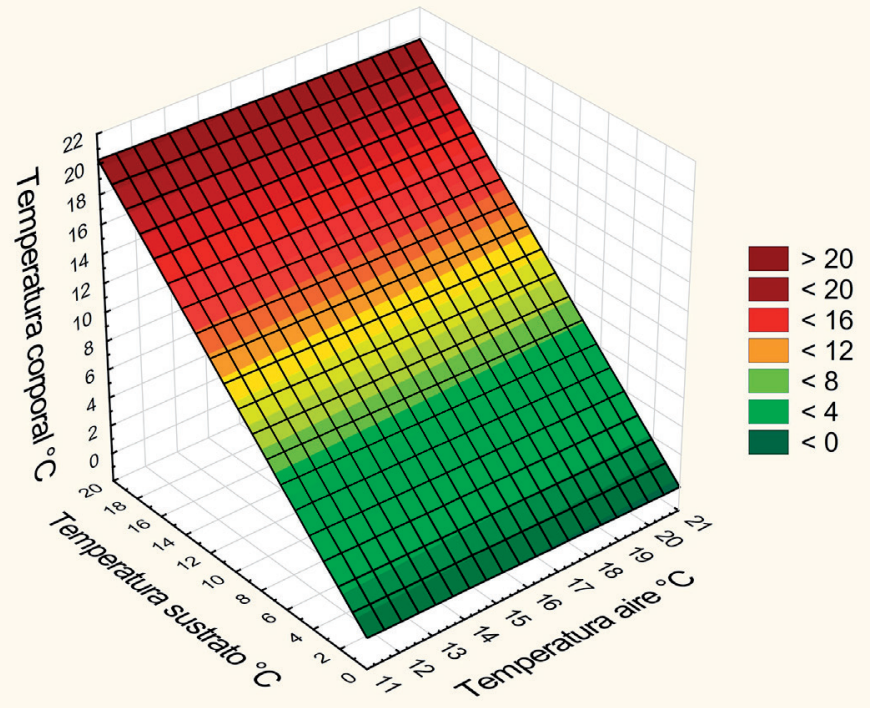

Figura 3. Relación entre la temperatura del aire ( $\mathrm{Ta})$, la temperatura del sustrato (Ts) y la temperatura corporal (Tc) de machos de $P$. thaul en condiciones de campo. / Relationship between air temperature (Ta), substrate temperature (Ts) and body temperature $(\mathrm{Tb})$ of $P$. thaul males under field conditions.

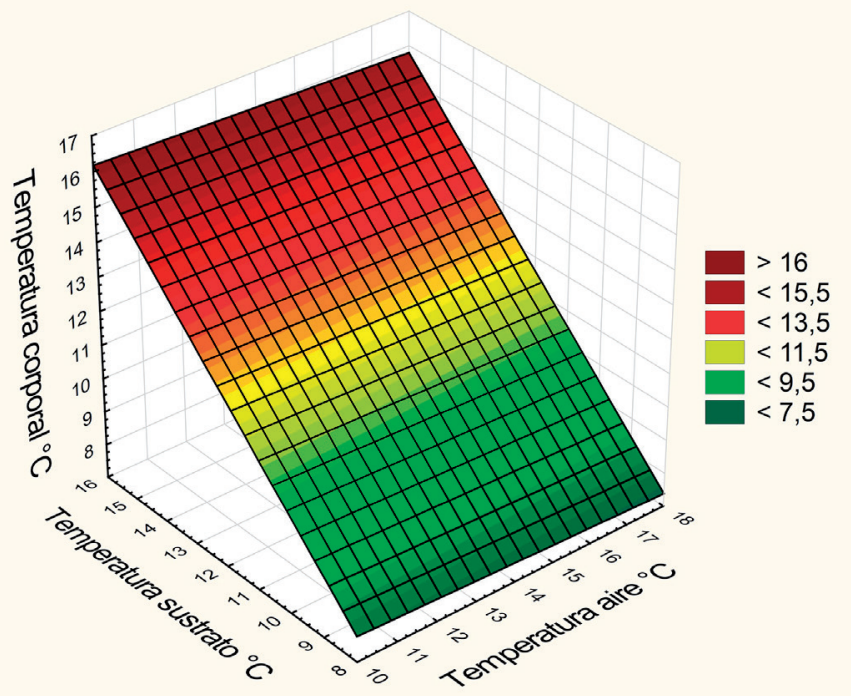

FIgURA 5. Relación entre la temperatura del aire (Ta), la temperatura del sustrato (Ts) y la temperatura corporal (Tc) de machos de $P$. thaul en condiciones de cautiverio durante la aclimatación de $10^{\circ} \mathrm{C}$. / Relationship between air temperature (Ta), substrate temperature (Ts) and body temperature (Tb) of $P$. thaul males in captive conditions during acclimation to $10^{\circ} \mathrm{C}$.
$0,4988 \mathrm{~T}_{\mathrm{a}}$ (Fig. 4) predice que los cambios producidos en la Tc dependerán de los cambios producidos en la temperatura de sustrato $\left(T_{s}\right)$ y en menor medida, de la temperatura del aire $\left(T_{a}\right)$.

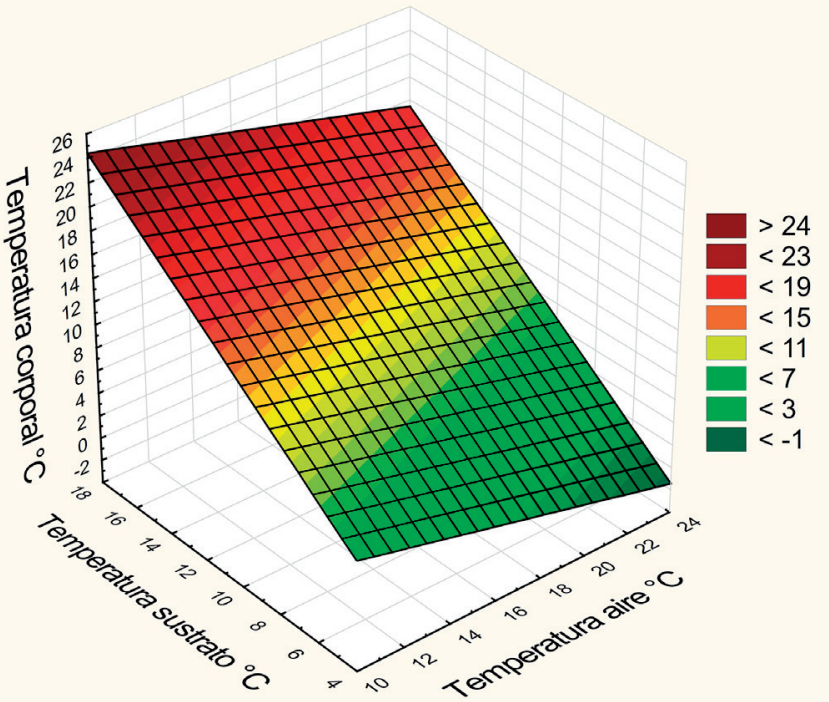

Figura 4. Relación entre la temperatura del aire (Ta), la temperatura del sustrato (Ts) y la temperatura corporal (Tc) de hembras de $P$. thaul en condiciones de campo. / Relationship between air temperature (Ta), substrate temperature (Ts) and body temperature (Tc) of $P$. thaul females under field conditions.

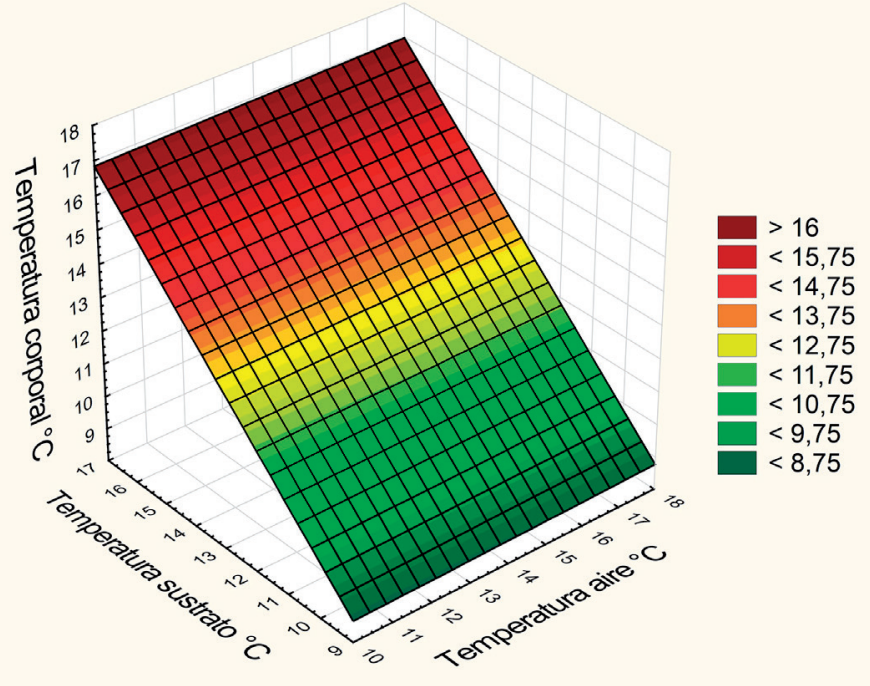

Figura 6. Relación entre la temperatura del aire (Ta), la temperatura del sustrato (Ts) y la temperatura corporal (Tc) de hembras de $P$. thaul en condiciones de cautiverio durante la aclimatación de $10^{\circ} \mathrm{C}$. / Relationship between air temperature (Ta), substrate temperature (Ts) and body temperature (Tb) of $P$. thaul females in captive conditions during acclimation to $10^{\circ} \mathrm{C}$. 
Situación similar se da en condiciones de laboratorio cuando fueron aclimatados a $10^{\circ} \mathrm{C} ; \mathrm{Tc}_{\text {machos }}=-0,1928+1,0526 \mathrm{~T}_{\mathrm{s}}-$ $0,047 \mathrm{~T}_{\mathrm{a}}$ (Fig. 5), $\mathrm{Tc}_{\text {hembras }}=-0,7533+1,0257 \mathrm{~T}_{\mathrm{s}}+0,0163 \mathrm{~T}_{\mathrm{a}}$ (Fig. 6) $\mathrm{y} 20^{\circ} \mathrm{C} \mathrm{Tc_{ \text {machos } }}=1,54+0,9277 \mathrm{~T}_{\mathrm{s}}+0,0011 \mathrm{~T}_{\mathrm{a}}$ (Fig. 7), $\mathrm{Tc}_{\text {hembras }}=$ $-0,1408+0,9705 T_{s}-0,025 T_{a}$ (Fig. 8). Además, el R ${ }^{2}$ obtenido (Tabla 2) indica que para las regresiones entre la temperatura corporal y la temperatura del sustrato hay una relación alta y significativa $\left(R^{2} \geq 0,867\right)$ y baja entre la temperatura corporal y la Ta $\left(R^{2} \leq 0,263\right)$.

Lo anterior revela que en condiciones de campo y cautiverio, la alta dependencia a la temperatura ambiental del sustrato caracterice tanto a machos y hembras de $P$. thaul como un ectotermo termoconformista, principalmente tigmotermo.

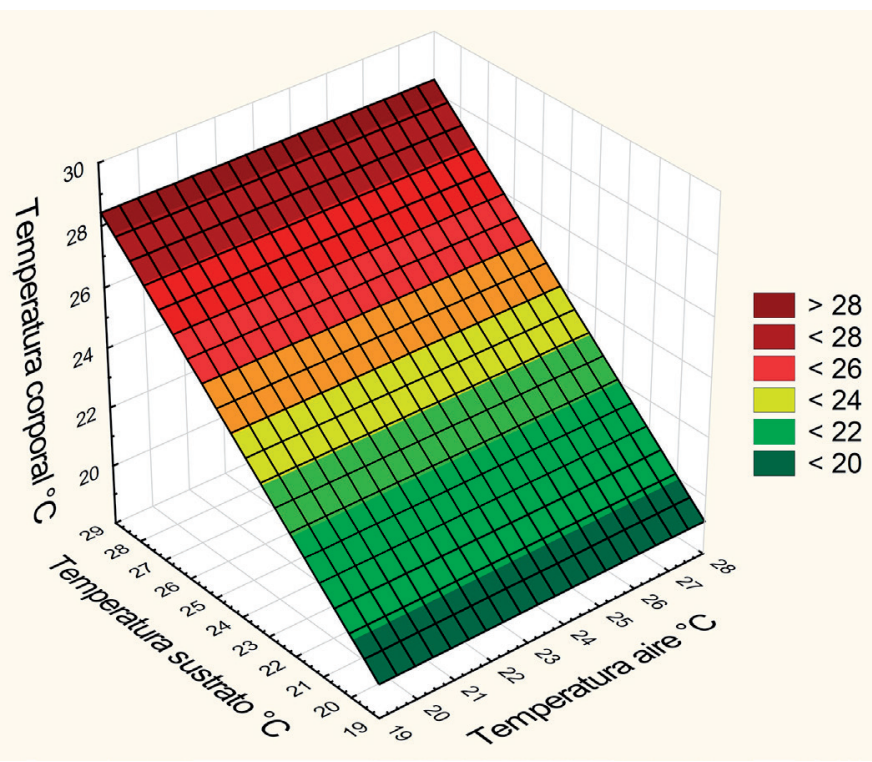

Figura 7. Relación entre la temperatura del aire (Ta), la temperatura del sustrato (Ts) y la temperatura corporal (Tc) de machos de $P$. thaul en condiciones de cautiverio durante la aclimatación de $20^{\circ} \mathrm{C}$. / Relationship between air temperature (Ta), substrate temperature (Ts) and body temperature $(\mathrm{Tb})$ of $P$. thaul males in captive conditions during acclimation to $20^{\circ} \mathrm{C}$.

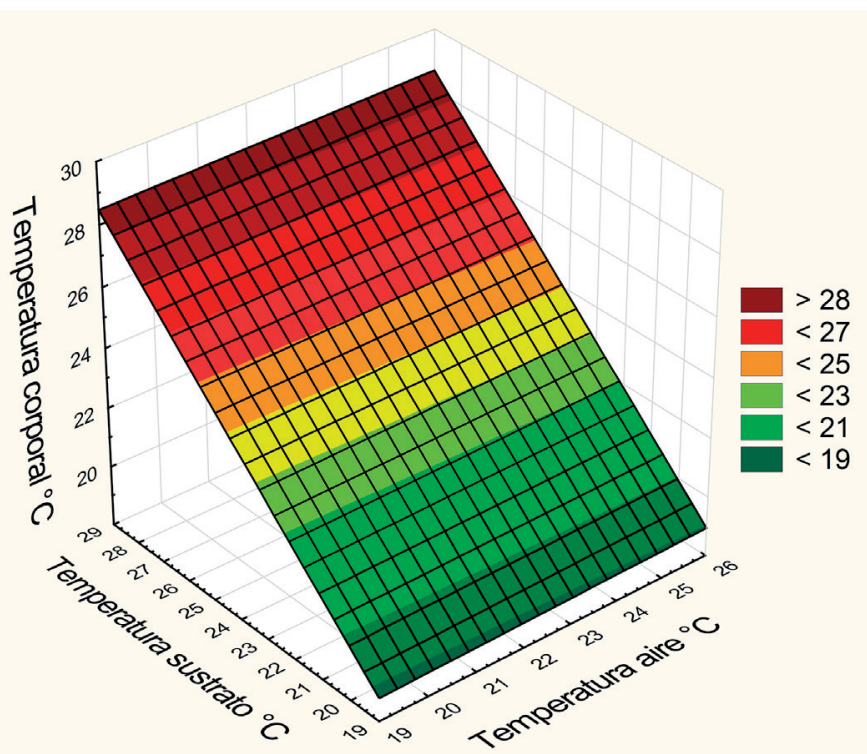

FiguRA 8. Relación entre la temperatura del aire (Ta), la temperatura del sustrato (Ts) y la temperatura corporal (Tc) de hembras de $P$. thaul en condiciones de cautiverio durante la aclimatación de $20^{\circ} \mathrm{C}$. / Relationship between air temperature (Ta), substrate temperature (Ts) and body temperature (Tb) of $P$. thaul females in captive conditions during acclimation to $20^{\circ} \mathrm{C}$.

TABLA 2. Valores de $R^{2}$ y $P$ de la pendiente de regresión lineal entre $T c$ y $T_{s}$ y entre $T c$ y $T_{a}$ para machos y hembras en condiciones de campo, aclimatación $10^{\circ} \mathrm{C}$ y aclimatación $20^{\circ} \mathrm{C}$. / $\mathrm{R}^{2}$ and $\mathrm{P}$ values of the linear regression slope between Tc and Ts and between Tc and Ta for males and females during the camp, acclimation $10^{\circ} \mathrm{C}$ and acclimation $20^{\circ} \mathrm{C}$.

\begin{tabular}{lccccc}
\hline Aclimatación & Sexo & $\mathbf{R}^{2} \mathbf{T}_{\mathrm{s}}$ & $\mathbf{P}$ & $\mathbf{R}^{2} \mathbf{T}_{\mathbf{a}}$ & $\mathbf{P}$ \\
\hline Campo & Machos & 0,9212 & $<0,05$ & 0,6642 & $<0,05$ \\
& Hembras & 0,9482 & $<0,05$ & 0,8992 & $<0,05$ \\
\multirow{2}{*}{$10^{\circ} \mathrm{C}$} & Machos & 0,8670 & $<0,05$ & 0,3300 & $<0,05$ \\
& Hembras & 0,8970 & $<0,05$ & 0,2630 & $<0,05$ \\
$20^{\circ} \mathrm{C}$ & Machos & 0,9110 & $<0,05$ & 0,3720 & $<0,05$ \\
& Hembras & 0,9130 & $<0,05$ & 0,3460 & $<0,05$ \\
\hline
\end{tabular}




\section{DISCUSIÓN}

Pleurodema thaul es la especie de anuro de más amplia distribución y mayor abundancia, dentro de Chile y Argentina. Esto ha llevado a que exista abundante información acerca de su biología, destacando un marcado dimorfismo sexual, el cual se expresa fundamentalmente en los tamaños corporales (Cei 1962). Sin embargo, los estudios realizados sobre esta especie han desechado un efecto de estos atributos corporales sobre la termoregulacion (Labra et al. 2008), probablemente debido a su pequeño tamaño (Iturra-Cid et al. 2014). De esta manera, para Iturra-Cid y colaboradores (2014) la alta dependencia con la temperatura ambiental que $P$. thaul presenta es una conducta termoconformista, tanto en condiciones de campo como de laboratorio, en la que la obtención de energía térmica se da principalmente mediante la tigmotermia (Iturra-Cid et al. 2014).

Los resultados de este estudio concuerdan con lo descrito para anfibios, donde esta conducta ha sido generalizada a muchas otras especies de este taxón (Castañeda et al. 2004, Vidal et al. 2008), algunas de ellas presentes en Chile, tales como Batrachyla taneniata Girard 1855 (Iturra-Cid \& Ortiz 2010), Rhinella spinulosa Wiegmann 1834 (Sinsch 1989, Lambrinos \& Kleier 2003, Alveal 2015) y Caliptocephalella gayi Duméril \& Bibron 1841 (Nova 2010). De esta manera, la conducta térmica se mantiene, independientemente de la temperatura de aclimatación a la cual son expuestos los individuos. Estas estrategias son apropiadas cuando los animales no necesitan invertir tiempo y energía seleccionando activamente microambientes (Huey \& Slatkin 1976, Huey 1982), situación dada en condiciones de cautiverio ya que la temperatura del medio suele mantenerse constante y en ausencia de oportunidades para la termorregulación (Labra \& Vidal 2003, Sanabria et al. 2011). Bajo la aclimatación a $20^{\circ} \mathrm{C}$ los machos se diferenciaron levemente con una media mayor de Tc que las hembras, lo que plantea que este comportamiento se asemeja con el de otros ectotermos juveniles de pequeño tamaño corporal, puesto que los machos, al ser más pequeños tienden a tener una temperatura corporal más alta que los individuos más grandes (Walguarnery et al. 2012, Zug et al. 2001), ya que individuos de menor tamaño intercambian energía térmica con el ambiente de forma más rápida, lo que aumenta la deshidratación y consecuentemente provoca un ascenso de la Tc (Zug et al. 2001, Sanabria et al. 2003, Sinervo et al. 2010). Sin embargo, no fue posible detectar la misma conducta con la Tc de campo y $T_{\text {sel }}$ ya que no se evidenciaron diferencias significativas entre los sexos.

Los resultados hacen presumir la idea de que ambos sexos utilizan una amplia gama de recursos térmicos cuando estos están disponibles (mediante un gradiente térmico), sobre todo cuando la humedad relativa no es un recurso limitante, ya que durante la experimentación se mantuvo constante esta variable dentro del sistema para evitar que influyera al momento de seleccionar una temperatura. Cabe destacar que las medias de $T_{\text {sel }}$ obtenidas en este estudio son superiores a lo descrito previamente por la literatura para esta especie (Iturra-Cid 2010, Ruiz-Aravena et al. 2014). Además no hubo un efecto significativo de la temperatura de aclimatación sobre este parámetro siendo coincidente con los resultados obtenidos por Ruiz-Aravena et al. 2014 en relación al efecto de la temperatura de aclimatación sobre la $\mathrm{T}_{\text {sel }}$ de una población $P$. thaul provenientes de un pequeño oasis en el desierto de Atacama.

Finalmente, el amplio rango de temperaturas seleccionadas por la especie se relaciona con los descrito por Bacigalupe y colaboradores (2018) y podría indicar que se trata de una especie generalista, lo que es común en ambientes heterogéneos (Stillman 2003), ya que la especie habita los medios con mayor variabilidad térmica, los cuales tienden a favorecer a los individuos que maximizan el rendimiento en un rango más amplio de temperaturas en comparación con ambientes más estables (Logan et al. 2014).

\section{CONCLUSIÓn}

$P$. thaul presentó una respuesta termoconformista en condiciones de campo y laboratorio, con una conducta principalmente tigmotérmica, en concordancia con la literatura. Las $T_{\text {sel }}$ para cada sexo en $P$. thaul no exhibieron diferencias significativas asociadas al dimorfismo sexual presente en la especie (hembras de mayor masa-tamaño que los machos). Sin embargo, se evidencia una media sobre los $24^{\circ} \mathrm{C}$, superior a lo descrito previamente por Iturra-Cid 2010 y Ruiz-Aravena et al. 2014. Ni la masa ni el tamaño asociados al sexo afectaron la termoconducta a nivel intersexual en $P$. thaul. Por último, las dos temperaturas de aclimatación en laboratorio $\left(10^{\circ} \mathrm{C}\right.$ y $\left.20^{\circ} \mathrm{C}\right)$ no influyeron sobre las $T_{\text {sel }}$ para la muestra.

\section{AGRADECIMIENTOS}

Los autores agradecen a Marta Briones por abastecer con moscas para la alimentación de los animales en cautiverio, Isadora Castro, Stefanie Fuentealba, Eric Beltrán, Pabla Hernández y Angelo Sandoval por ayudar a capturar los individuos en terreno; a Marcela Vidal por sus sugerencias en el manuscrito y a Conicyt Becas Doctorado Nacional 201921191272 


\section{REFERENCIAS}

Alveal, N. 2015. Relaciones entre la fisiología térmica y las características bioclimáticas de Rhinella spinulosa (Anura: Bufonidae) en Chile a través del enlace mecanicista de nicho térmico. Tesis de Magister, Universidad de Concepción, Concepción, Chile.

Alveal, N., Díaz-Páez, H., Ortiz, J.C. 2016. Thermal tolerance in the Andean toad Rhinella spinulosa (Anura: Bufonidae) at three sites located a long a latitudinal gradient in Chile. Journal of Thermal Biology 60: 237-245.

Angilletta, M., Niewiarowskib, P., Navas, C. 2002. The Evolution of Thermal Physiology in ectotherms. Journal of Thermal Biology 27(4): 249-268.

Bartholomew, C. 1982. Physiological control of body temperature. En: Gans, C., Pough, F. (Eds) Biology of Reptilia 12: 167211. Academic Press, London, New York, Paris, San Diego, San Francisco, São Paulo, Sydney, Tokyo, and Toronto.

Castañeda, L., Lardies, M., Bozinovic, F. 2004. Adaptive latitudinal shifts in thermal traits of a terrestrial isopod. Evolutionary ecology research 6: 570-593.

Cei, J.M. 1962. Batracios de Chile. Ediciones de la Universidad de Chile. Santiago. 128 pp.

Francino, E. 2011. Umbral de intensidad acústica que evoca respuesta en hembras del anuro Pleurodema thaul. Tesis de Grado, Universidad de Chile, Santiago, Chile.

Gilchrist, G. 1995. Specialist and generalist in changing environments. I. Fitness landscape of thermal sensitivity. The American Naturalist 146: 252-270.

Hillman, S. 2009. Ecological and environmental physiology of amphibians. Oxford University Press. Oxford. 469 pp.

Huey, R. 1982. Temperature, physiology, and the ecology of reptiles. En: Gans, C., Pough, F.H. (eds) Biology of Reptilia 12: 25-91. Academic Press, London, New York, Paris, San Diego, San Francisco, São Paulo, Sydney, Tokyo, and Toronto.

Huey, R. 1991. Physiological consequences of habitat selection. American Naturalist 137: 91-115.

Huey, R., Slatkin, M. 1976. Cost and benefits of lizard thermoregulation. The Quarterly Review of Biology 51: 363-384.

Hutchison, V., Hill, L. 1977. Thermal selection of bullfrog tadpoles (Rana catesbeiana) at different stages of development and acclimation temperaturas. The Journal of Thermal Biology 3: $57-60$

Iturra-Cid, M., Ortiz, J. 2010. Batrachyla taeniata (NCN). Body temperatura. Herpetological Review 41: 331-333.

Iturra, C.M., Vidal, M., Labra, A., Ortiz, J. 2014. Winter Thermal Ecology Of Pleurodema thaul (Amphibia: Leptodactylidae). Gayana 78 (1): 25-30.
Labra, A., Vidal, M. 2003. Termorregulación en reptiles: Un pasado veloz y un futuro lento. Ediciones Universidad Católica de Chile, 207-224.

Labra, A., Vidal, M., Solis, R., Penna, M. 2008. Ecofisiología de Anfibios y reptiles. En: Vidal, M., Labra, A. (Eds) Herpetología de Chile Santiago: Science Verlag, 483- 516.

Lambrinos, J., Kleier, C. 2003. Thermoregulation of juvenile Andean toads (Bufo spinulosus) at $4300 \mathrm{~m}$. Journal of Thermal Biology 28: 15-19.

Lillywhite, B. 1971. Temperature selection by the bullfrog Rana catesbeiana. Comparative Biochemistry and Physiology A 40: 171-213.

Logan, M.L., Cox, R.M., Calsbeek, R. 2014. Natural selection on termal performance in a novel thermal environment. Proceedings of the National Academy of Sciences of the United States of America 19: 1-5.

Nova, R. 2010. Proyecciones sobre el estudio de la termofisiología de Calyptocephalella gayi en Chile y los impactos del cambio climático. Tesis de Grado, Universidad del Biobío, Chillán, Chile.

Pörtner, H. 2002. Climate variation and physiology basis to temperature dependent biogeography: systemic to molecular hierarchy of thermal tolerance in animals. Comparative Biochemistry and Physiology 132: 739-761.

Pough, F., Gans, C. 1982. The vocabulary of reptilian thermoregulation. En: Gans, C., Pough, F. (Eds) Biology of reptilia 12: 17-23. Academic Press, London, New York, Paris, San Diego, San Francisco, São Paulo, Sydney, Tokyo, and Toronto.

Riveiro Da Luz, A.M., Alvarenga Álvares, B. 1998. Física general con experimentos sencillos (4a edición.). Oxford University Press, México.

Sanabria, E.A., Quiroga, L.B., Acosta, J.C. 2003. Ecología térmica de una población de Bufo arenarum (anura: Bufonidae) en un humedal del centro-oeste de argentina. Boletin de la Sociedad Herpetológica Mexicana 11(2): 33-41.

Sanabria E., Quiroga, L., Martino, A. 2011. Seasonal changes in the thermoregulatory strategies of Rhinella arenarum in the Monte desert, Argentina. Journal of Thermal Biology 36: 23-28.

Sinervo, B., Méndez de la Cruz, F., Miles, D., Heulin, B., Bastiaans, E., Villagran, M., Lararezendiz, R., Martinez-Méndez, N., Calderon, M., Meza, R., Gadsden, H., Avila, L., Morando, M., De la riva, I., Victoriano P., Duarte C., Ibargúengoytía N., Aguilar C., Massot M., Lepetz V., Oksanen, T., Chapple D., Bauer A., Branch W., Clobert J., Sites J. 2010. Erosion of lizard diversity by climate change and altered termal niches. Science 328: 894-899.

Sinsch, U. 1989. Behavioural thermoregulation of the Andean toad (Bufo spinulosus) at high altitudes. Oecologia 80: 32-38. 
Ruiz-Aravena, M., Gonzalez-Mendez, A. Estay, A., GaitanEspitia, J. Barria-Oyarzo, I. Bartheld, J. Bacigalupe, L. 2014. Impact of global warming at the range margins: phenotypicplasticity and behavioral thermoregulation will buffer anendemic amphibian. Ecology and Evolution 4(23): 4467-4475.

Stillman, J. 2003. Acclimation capacity underlies susceptibility to climate change. Science 301: 65.

Vidal, M., Ortiz, J., C., Labra, M., A. 2008. Intraspecific variation in a physiological thermoregulatory mechanism: the case of the lizard Liolaemus tenuis (Liolaeminae). Revista Chilena de Historia Natural 81: 171-178.
Vidal, M., Novoa-Muñoz, F., Werner, E., Torres, C., Nova, R. 2017. Modeling warming predicts a physiological threshold for the extinction of the living fossil frog Calyptocephalella gayi. Journal of Thermal Biology 69: 110-117.

Walguarnery J., Goodman R., Echternacht, A. 2012. Thermal biology and temperature selection in juvenile lizards of co-occurring native and introduced anolis species. Journal of Herpetology, 46(4): 620-624.

Zug, G., Vitt, L., Caldwell, G. 2001. Herpetology. An introductory biology of amphibians and reptiles. Academy Press. New York, USA.

Received: 05.11.2018

Accepted: 24.04.2019 